\title{
USO Y DIFUSIÓN DEL FRIJOL DE ABONO (Mucuna deeringiana) EN LAS LADERAS DEL LITORAL ATLÁNTICO DE HONDURAS1
}

\author{
Daniel Buckles ${ }^{2}$, Ignacio Ponce ${ }^{3}, J$ Gustavo Sain ${ }^{4}$ y Gilmer Medina ${ }^{5}$
}

\begin{abstract}
RESUMEN
Uso y difusión del frijol de abono (Mucuna deeringiana) en las laderas del litoral Atlántico de Honduras. La conservación del suelo, los nutrimentos y el agua son los principales problemas que afrontan los agricultores e investigadores agrícolas en los sistemas de cultivo basados en el maíz en Centroamérica y México. Algunos productores de Honduras han ideado y refinado una tecnología eficaz para el cultivo del maíz en ladera en la que se siembra el frijol de abono (Mucuna deeringiana) en rotación con el maíz de postrera (o temporada seca). Los resultados de la encuesta realizada en el Departamento de Atlántida en Honduras indican que el 66\% de los agricultores en ladera siembran la mayor parte de su maíz de postrera a través del colchón protector que forma el frijol de abono durante la época de lluvia. Entre las ventajas de esta rotación en comparación con el cultivo tradicional de roza y quema, figuran mayores rendimientos con menos dependencia de insumos externos, períodos de descanso más breves, costos más bajos de mano de obra para preparar del terreno, menos erosión del suelo y menos riesgo de que haya daños por sequía durante la postrera. Los problemas vinculados con la difusión del sistema incluyen el riesgo de que haya derrumbes en las laderas más empinadas y una mayor incidencia de plagas (ratas) en el maíz que se siembra en el mantillo de la abonera. Lo más usual es que la cantidad de tierra disponible en los mercados regionales de tierra sea el factor que determina la difusión de la tecnología, no el tamaño de la finca ni las formas de tenencia de la tierra.
\end{abstract}

\begin{abstract}
Use and diffusion of bean (Mucuna deeringiana) as green manure on the hills of the Atlantic Coast in Honduras. The soil, nutrient and water conservation is the main problem faced by farmers and investigators under the corn crop-based systems in Central America and Mexico. Several farmers from Honduras have come up with an efficient technology for the corn planted on hills, in which the bean manure is planted in rotation with corn during the dry season. The results from the conducted survey, at the Departamento de Atlantida in Honduras, indicate that $66 \%$ of the farmers grow most of the corn through the protective mat produced by the beans during the rainy season. Among the advantages of this rotation, compared to the traditional burned and cleared land, are the higher yields with les ser dependency of external imputs, shorter rest periods, lower land preparation costs, less soil erosion and lower risk of drought damages. The problems linked to the diffusion ofthe system include the risk ofland slides and a higher plague's incidence (rats) on the corn planted through the manure mat. Usually, the amount of land available in the regionalland markets is the determining factor on the diffusion of this system, and not the size of the farm nor the form of land tenancy.
\end{abstract}

\section{INTRODUCCIÓN}

El problema más apremiante que enfrentan los productores e investigadores del maíz en los sistemas agrícolas basados en ese cultivo en Centroamérica y México, es la conservación del suelo, los nutrimentos y el agua. Los factores sociales y técnicos, que contribuyen a crear los problemas relacionados con el manejo de los recursos naturales, son complejos y a menudo abarcan distintos contextos sociales y sistemas de cultivo. En muchas partes del

\footnotetext{
1 Presentado en la XXXIX Reunión Anual del PCCMCA en Guatemala, América Central.

2 Antropólogo, CIMMYT.

3 Sociocconomista, SRN.

4 Economista, CIMMYT.

5 Agrónomo, SRN.
} 
trópico húmedo, estos factores incluyen el desplazamiento del cultivo de los granos básicos hacia laderas empinadas debido a que los terrenos aptos para el mismo están ocupados por cultivos de plantación y la explotación ganadera extensiva. Estas industrias en muchos casos están orientadas hacia la exportación o el abasto de las necesidades de consumo de los grupos sociales más acomodados. Por otra parte, el crecimiento demográfico y los movimientos migratorios ejercen una presión cada vez mayor en los recursos naturales tanto en tierras que ya son intensamente cultivadas como en zonas fronterizas. Estas fuerzas sociales generales suelen dar por resultado sistemas agrícolas no sostenibles que se caracterizan por períodos de descanso muy cortos o nulos, la quema frecuente del rastrojo, la invasión de malezas gramíneas y la erosión. En casi todo el trópico húmedo de Centroamérica y México, el descanso tradicional ha dejado de cumplir su función de ayudar a rec;uperar la fertilidad del suelo, protegerlo de la erosión y reducir la incidencia de malezas, lo que ha dado como resultado pérdidas de suelo irreversibles y reducciones fuertes en la productividad agrícola.

Pese a la extrema pobreza y los bajos niveles de educación formal, los agricultores no han permanecido pasivos ante los problemas de la degradación de los recursos naturales. Durante más de 40 años, indígenas del Departamento de Isabal en Guatemala y el sur de México (Veracruz y Tabasco) generaron y refinaron una tecnología que se basa en una, leguminosa conocida como frijol terciopelo, nescafé o frijol de abono (Mucuna deeringiana). Estos productores crearon, con frecuencia en forma independiente, prácticas semejantes de manejo de la fertilidad, malezas yagua, ensayándolas lenta pero seguramente y difundiéndolas ellos mismos. Hasta la fecha, las investigaciones científicas de las propiedades del frijol de abono y de su adopción no han podido sostener el ritmo de su amplia aceptación. En el presente trabajo se examina el uso y la difusión del frijol de abono en las laderas del Litoral Atlántico de Honduras, con el fin de identificar las características de esta tecnología y los factores que contribuyen a su adopción.

\section{METODOLOGIA}

El presente estudio es resultado de una investigación efectuada conjuntamente por la Secretaría de Recursos Naturales (SRN), el Proyecto Desarrollo del Bosque Latifoliado (PDBL) y el Centro Internacional de Mejoramiento de Maíz y Trigo (CIMMYT). La investigación sobre el frijol de abono se inició en 1982, con un estudio de diagnóstico del maíz en el municipio de Jutiapa (PNIACIMMYT, 1983). En aquel momento, los investigadores observaron que un número cada vez mayor de agricultores de ladera sembraban el frijol de abono en rotación con el maíz. Para fines del decenio de 1980 se inició una serie de investigaciones identificadas como prioritarias por la Dirección Agrícola Regional del Litoral Atlántico y que fueron auspiciadas por el Programa Regional de Maíz para Centro américa y El Caribe (PRM) (Duron, 1989). En 1990 y 1991 se realizaron varios sondeos sobre el uso del frijol de abono, así como una encuesta exploratoria dirigida a determinar el grado de difusión de la tecnología y los factores que influyen en la adopción (Buckles, 1990 y Buckles et al. 1991). Estos estudios facilitaron la realización de una encuesta formal en la región, que se inició en febrero de 1992 con la colaboración de la Dirección Agrícola Regional del Litoral Atlántico en La Ceiba, las Agencias de Desarrollo en Tela y en Jutiapa, la Dirección Agrícola Regional de San Pedro Sula,el Proyecto Desarrollo del Bosque Latifoliado (PDBL) en La Ceiba, el Centro Universitario Regional del Litoral Atlántico (CURLA) y el Centro Internacional de Mejoramiento de Maíz y Trigo (CIMMYT).

La zona del presente estudio abarca solamente las laderas, donde están ubicados la mayoría de los agricultores de maíz, que se consideran un sector social prioritario. Unas 6,400 familias, que juntas incluyen a cerca de 41,000 personas, viven en las comunidades enclavadas en las laderas del Departamento de Atlántida. Los datos presentados en este informe se derivaron de una encuesta de 128 familias en 16 aldeas, 11 en la zona de Jutiapa y cinco en la zona de Tela. 
El cuestionario fue evaluado y revisado, los encuestadores participaron en un taller de capacitación y cada cuestionario fue revisado por los coordinadores de la encuesta al final de cada día. La encuesta se llevó a cabo en un período de tres semanas.

\section{GENERALIDADES SOBRE LA REGION}

\section{Medio ambiente}

El Departamento de Atlántida comprende dos grandes zonas agroecológicas, las planicies de la costa y las laderas de la cordillera Nombre de Dios, que ocurren en forma paralela a la planicie costera. La región tiene un clima cálido-humedo con una distribución de lluvias bimodal que oscila entre 2,000 y $3,300 \mathrm{~mm}$, condiciones adecuadas para una vegetación primaria de bosque tropical húmedo. La temperatura anual media en la zona de ladera $\left(26^{\circ} \mathrm{C}\right)$ es más baja que en la zona plana $\left(28^{\circ} \mathrm{C}\right)$ y la distribución de las lluvias está sujeta a una mayor variación por efecto de los microclimas. La planicie está caracterizada por suelos de origen aluviales, en su mayoría de buena fertilidad. En contraste, las laderas están constituidas por materiales de origen sedimentario, aunque existen áreas en donde predominan materiales de origen ígneo y metamórfico s que dan origen a suelos poco evolucionados, relativamente delgados y de permeabilidad lenta. La zona de ladera es quebrada, con pendientes que varían del 10 al más del $100 \%\left(45^{\circ}\right)$.

\section{El sistema de finca}

El año agrícola se divide en dos temporadas, la primera o primavera (siembra en junio y cosecha en noviembre) y la postrera o verano (siembra en diciembre y cosecha en abril). Debido a las abundantes lluvias y altas temperaturas es posible sembrar una gran variedad de cultivos. Sin embargo, el uso de la tierra en la zona de planicie, dedicada principalmente a cultivos de exportación, como la piña y el banano, y la ganadería lechera, es marcadamente distinto del de la zona de ladera en la que predomina el maíz, con otros cultivos secundarios como fri- jol común, yuca, arroz, cacao y café. Tradicionalmente, la primera ha sido la temporada agrícola más importante en lo que a la siembra del maíz se refiere, aunque el auge del sistema de abonera ha realzado la importancia de la postrera en el área dedicada al maíz, como se verá en detalle a continuación.

Los bosques húmedos que caracterizan la región han sido gravemente afectados por la tala, el cultivo ambulatorio y la expansión de la industria ganadera hacia la zona de ladera. No obstante, la región sigue siendo fronteriza (o sea, que la zona de cultivo puede expandirse por estar junto a áreas forestadas). En primer lugar, muchas laderas todavía están cubiertas por vegetación secundaria (guamil) derivada principalmente de especies forestales. El hecho de que todavía hay tierra sin cultivar permite el cultivo ambulatorio, el cual a menudo es seguido por el pastoreo de ganado vacuno durante varios años hasta que finalmente la tierra vuelve a ser cubierta por vegetación secundaria. En segundo lugar, grandes extensiones de tierra en la zona de ladera pertenecen a ganaderos que viven en centros urbanos en la planicie costera. Los pequeños agricultores pueden alquilar estas tierras mediante contratos de arrendamiento, generalmente por una suma modesta, siempre que la vuelvan a dedicar a los pastos cuando se agote su potencial agrícola. Por estas razones, los productores de la región tienen acceso relativamente fácil a tierras para sembrar maíz y en promedio el tamaño de estas fincas es mucho mayor que en las regiones más intensamente cultivadas de Honduras, como se describe en seguida.

El tamaño o área de finca se refiere al total de tierras ocupadas con cultivos ya sea anuales o perennes, con pastos naturales o artificiales, o que hayan estado en descanso durante el año agrícola 1991-1992. Como tal, esta variable comprende aquellas parcelas trabajadas en calidad de arrendamiento o prestadas durante ese período pero no comprende el área del solar o patio. Definidas de esta manera, el promedio del tamaño de las fincas en el Litoral Atlántico de Honduras es de 11 manzanas (mz) campesinas ( 9,2 hectáreas). ${ }^{9}$ Sin embargo, el $60 \%$ de las fincas del área se concentra en el intervalo que va de 0 hasta $6 \mathrm{mz}$ (Cuadro 1). Se observa también que existe un grupo 
Cuadro 1. Distribución del área de la finca.

\begin{tabular}{lcc}
\hline Area $(\mathbf{m z}) *$ & $\begin{array}{c}\text { No. de } \\
\text { Agricultores }\end{array}$ & $\%$ \\
\hline$<2$ & 32 & 25 \\
$2-4$ & 29 & 23 \\
$4-6$ & 16 & 12 \\
$6-8$ & 9 & 7 \\
$8-10$ & 7 & 6 \\
$10-12$ & 5 & 4 \\
$12-14$ & 4 & 3 \\
$>14$ & 26 & 20 \\
\hline Total & 126 & 100 \\
\hline
\end{tabular}

* Los datos del área están presentados en manzanas campesinas, con un coeficiente de conversión de manzana campesina a metros de $8,365 \mathrm{~m}^{2}$.

relativamente importante de fincas "grandes", es decir, con más de 14 mz, el cual agrupa al 20\% de los agricultores.

\section{RESULTADOS Y DISCUSION}

\section{El sistema de abonera}

La abonera es un campo sembrado con frijol de abono en monocultivo que luego es rotado con maíz de postrera (Figura 1). La abonera se establece por primera vez en un campo haciendo una siembra intercalada de la leguminosa entre 40 y 55 después de la siembra de maíz de postrera (de mediados a fines de febrero). Se siembran entre los surcos de maíz dos o tres semillas por postura a una distancia de aproximadamente un metro. El maíz de postrera se cosecha entre marzo y abril, y la leguminosa se deja crecer sola durante la primera. El maíz de primera se siembra en un campo distinto siguiendo la tecnología tradicional de roza, tumba y quema; mientras tanto, el frijol de abono desarrolla una densa mata vegetativa de 1,5 metros de altura. La etapa vegetativa de la leguminosa termina a fines de noviembre, cuando se forma la semilla. Los agricultores entonces cortan la mata y, unas cuantas semanas después, siembran el maíz de postrera con chuzo a través del colchón de hojas y tallos cn descomposición. Las dos terceras partes de los agricultores entrevistados indicaron que dejan que la abonera se autosiembre y los demás afirmaron que cada año resiembran el frijol de abono en los campos de maíz de postrera. Los productores señalaron también que se necesitan tres años para que se establezca una abonera que no requiere que se le resiembre y que brinda el máximo beneficio al maíz de postrera.

La abonera es una tecnología de usos múltiples. Por una parte, facilita grandemente el chapeo del terreno, ya que el frijol de abono es una leguminosa agresiva que ahoga casi todas las malezas que con ella compiten y es muy fácil de cortar. El mantillo que forma conserva la humedad del suelo y lo protege de la erosión; además, las hojas, tallos y raíces en descomposición proporcionan nutrimentos al siguiente cultivo de maíz. Como observó un agricultor hondureño: "La tierra cobarde se vuelve valiente" cuando se le siembra con el frijol de abono.

\section{Varias medidas de adopción}

Los agricultores guatemaltecos y hondureños que emigraron de la zona fronteriza introdujeron el sistema de abonera en la costa norte de Honduras a principios de los 1970. Nosotros estimamos que para el año 1992, aproximadamente el $66 \%$ de los agricultores en ladera en el Departamento de Atlántida sembraban al menos parte de su maíz en aboneras. Fue similar el nivel de adopción (61 \% de los agricultores encuestados) estimado con base en una encuesta realizada en 1990 en esa misma región (Buckles, 1990). En 1992, el 34\% de los encuestados indicaron que no sembraron su maíz de postrera del año en curso en aboneras, pero cerca del $50 \%$ de ellos habían usado aboneras en algún momento en el pasado reciente. Por consiguiente, se estima que el $83 \%$ de los productores en el área de estudio (en total, más de 5,250 agricultores en ladera) tienen experiencia directa con la tecnología. 


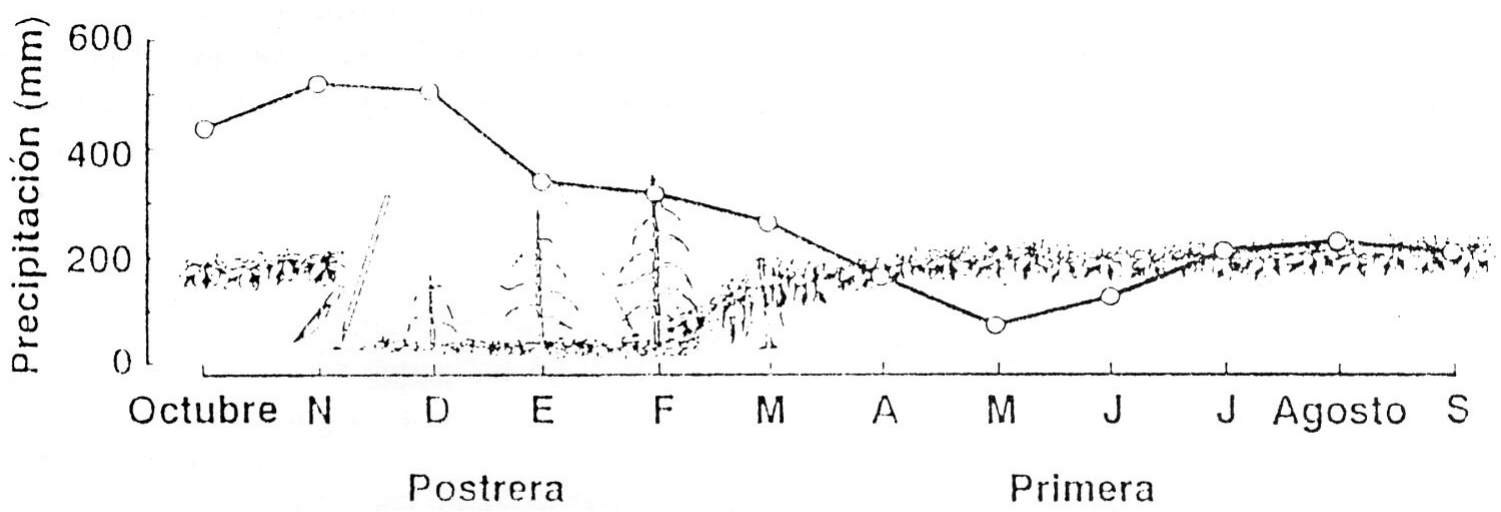

Figura 1. El sistema de abonera y preciptación anual media (1980-89), La Ceiba, Honduras.

Entre muchos adoptadores, el uso de la tecnología es casi completa. En 1992, aproximadamentc el 55\% de los adoptadores cullivaron todo su maíz de postrera en aboneras, en tanto que el $78 \%$ de cllos sembraron más de la. mitad de su maíz de postrera en esta forma. Por otra parte, la conversión al sistema parece ser inmediata y uniforme, situación poco común en la adopción de nuevas tecnologías. El análisis de regresión de los datos de la encuesta reveló que no existe relación alguna entre el porcentaje del maíz de postrera sembrado en abonera y el número de años de experiencia con la tecnología. Al parecer, los adoptadores con menos de cinco años de experiencia siembran en aboneras igual cantidad de su maíz de postrera que los usuarios con más experiencia.

A nivel regional, el sistema de abonera ha adquirido una importancia considerable en el sector maíz. Durante la postrera de 1991, en un 55\% del área cultivada con maíz se sembraron aboneras, las cuales produjeron el $65 \%$ de la cosecha total.

La gran proporción de los agricultores en ladera que cultivan el maíz de postrera en abonera, el uso extenso de la tecnología y la aporlación total de este sistema al sector maíz de la región, constituyen una fuerte indicación del grado de adopción de la tecnología. La magnitud del cambio tecnológico reflejada en la difusión del sistema es aun más evidente si se considera que hace 20 años no se producía maíz en esta forma en la región. En la siguiente sección se presentan los patrones de adopción a través del espacio y del tiempo, que serán examinados con el fin de identificar las condiciones que se determinaron.

\section{Palrones de adopción en el espacio y el tiempo}

La región tiene una superficie de $3,300 \mathrm{~km}^{2}$ en ladera entre los municipios de Jutiapa y Tela. Las tasas de adopción varían poco en la región: según las encuestas de 1990 y 1992, Tela tiene tasas un poco más altas de adopción y de número promedio de años de uso de la tecnología, pero las diferencias con otros municipios de la región no son estadísticamente significativas. Sin embargo, encuestas en la región sugirieron que algunas comunidades en el municipio de Tela fueron el primer foco de adopción generalizada dentro del Departamento de Atlántida.

Los informes de los agricultores acerca de dónde y cuándo observaron por vez primera la tecnología, indican que hubo un proceso de difusión desde Guatemala y los departamentos hondureños de Cortés, Santa Bárbara y Copán hacia el sureste del país hasta llegar a Atlántida y otras regiones. Los primeros adoptadores (más de 10 años de experiencia), citaron el área costera fronteriza entre 
Guatemala y Honduras como su primer punto de contacto con la tecnología, en tanto que los que la adoptaron después señalan comunidades vecinas en el mismo Departamento o las suyas propias. Esta información es congruente con lo indicado por los agricultores en numerosas entrevistas, es decir, que la tecnología se originó en Guatemala y se difundió hacia la costa norte de Honduras.

La Figura 2 muestra los niveles acumulativos de adopción en Atlántida entre 1977 y 1992, según el año de adopción que recuerdan los agricultores. Estos niveles han sido ajustados para excluir en cualquier año dado a los agricultores que cran demasiado jóvenes (menos de 20 años) para ser jefes de familias o que en ese tiempo vivían fuera del Departamento. Este último factor es particularmente importante, ya que muchos agricultores que ahora residen en Atlántida llegaron desde otras partes del país en donde quizá habían adoptado la tecnología. El proceso de adopción en Atlántida empezó lentamente, aumentó rápidamente durante los 1980 y se niveló en los últimos años. Es evidente, a partir de los datos de la encuesta y de las entrevistas, que el sistema de abonera es relativamente reciente entre las opciones de cultivo que existen en la región. Los primeros informes de su uso datan del comienzo los años 1970. Aunque el pequeño número de casos de adopción durante esa década no permite seguir el avance de la adopción, al parecer durante los 10 años después de la introducción de la tecnología en la región, la adopción fue muy lenta. En 1980, sólo e $15 \%$ de los agricullores sembraban maíz de postera en aboneras. En el decenio de 1980 hubo una tasa de adopción muy elevada: 5\% anual hasta llegar a más del $60 \%$ en 1990. En los últimos años, el avance de la adopción se ha nivelado considerablemente, lo cual plantea interrogantes acerca de los factores que obstaculizan la adopción en la región, Las siguientes secciones del informe examinan los factores qne condicionaron el patrón de difusión observado en el Departamento de Atlántida.

\section{La evaluación campesina}

En esta sección exploramos las opinioncs de los agricultores con respecto a las características distintivas de la tecnología. Durante la encuesta, se pidió a los agricultores que definieran la importancia relaliva de las características distintivas de la tecnología que fueron

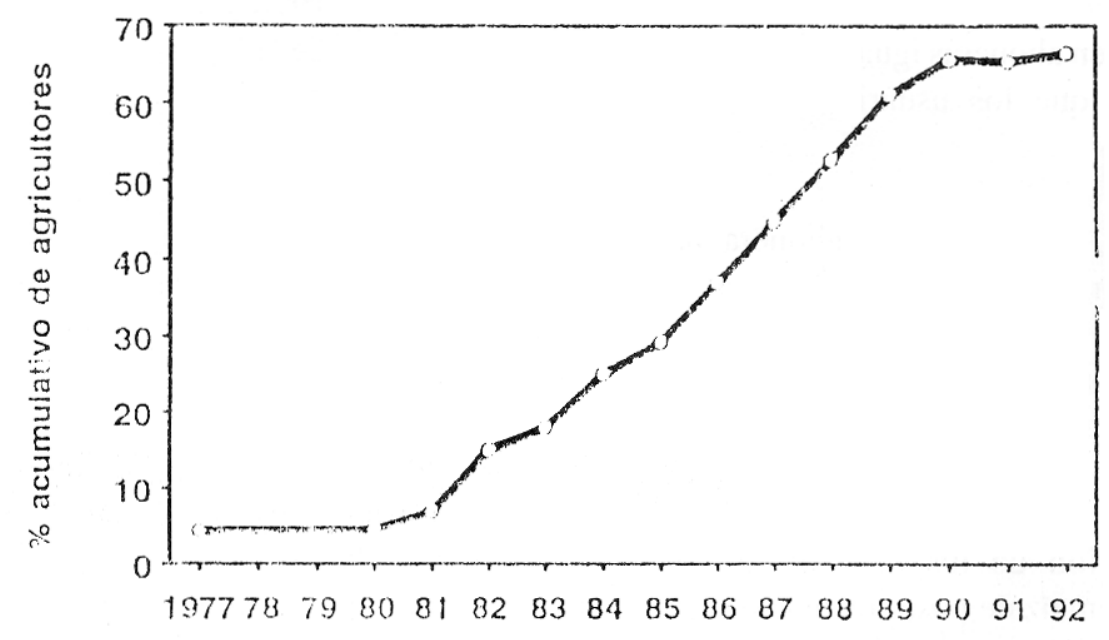

Figura 2. Porcentaje acumulativo de agricultores que adoptaron el sistema de abonera (ajustado según la edad y antecedentes migratorios del agricultor). 
identificadas en sondeos anteriores. Los aspectos positivos de la tecnología que citaron incluyeron el suministro de nutrimentos (abono) al maíz, el control de male-zas, facilidad de la preparación del terreno, conservación del agua y control de erosión. Entre las desventajas que mencionaron figuran que las aboneras crean condiciones propicias para las plagas (en especial, ratas y víboras venenosas), que pueden provocar derrumbes en laderas muy empinadas y que, cuando se les utiliza en rotación, producen una sola cosecha de maíz al año, cuando sin la abonera se pueden producir dos.

Se utilizaron medios visuales para ayudar a los agricultores a poner en orden de prioridad las distintas ventajas y desventajas de las aboneras. Se les mostraron tarjetas que ilustran las características y se les pidió su primera y segunda selección tanto para las ventajas como las desventajas. Un análisis de las opiniones exteriorizadas sobre la importancia relativa de estas características permitirá elucidar cuáles son los'criterios en que se basan los agricultores para adoptar la tecnología.

Cerca del $40 \%$ de los adoptadores encuestados en 1992 opinan que la característica más importante de la abonera es el abono natural que ésta proporciona al cultivo (Cuadro 2). Aproximadamente el $23 \%$ señaló la facilidad de la preparación de la tierra como el aspecto más relevante de la tecnología, mientras que el $21 \%$ resaltóla conservación de la humedad del suelo como la característica más importante de todas. El 16\% restante clasificó por partes iguales el control de malezas y de la erosión como el aspecto más importante. Aunque esta sencilla evaluación revela el alto valor que se le asigna al efecto fertilizante de las aboneras, la gran variedad de respuestas resalta los numerosos usos que éstas tienen desde el punto de vista de los agricultores.

Una recopilación de la segunda característica más importante de la tecnología revela la relevancia de la mano de obra ahorrada en comparación con otros aspectos. $\mathrm{Si}$ bien los efectos fertilizantes siguen manteniendo su elevada clasificación, la facilidad de la preparación de la tierra y el control de malezas, ambos efectos que ahorran mano de obra, representan más de la mitad de las respuestas.

Aunque la alta aceptación de la tecnología y la identificación explícita de sus principales características indican su popularidad entre los agricultores, los datos de la encuesta revelan algunas limitaciones importantes (Cuadro 3). El $46 \%$ de los usuarios encuestados señalaron que las plagas y, en particular, las ratas les preocupaban más en las parcelas con aboneras que en las que no las tienen. La cobertura creado por la abonera es un ambiente protegido atractivo para las ratas, aunque muchos agricultores reconocieron que las fluctuaciones cíclicas de las poblaciones de roedores influyen más en la gravedad del problema.

Aproximadamente el $28 \%$ de los agricultores mencionaron que los derrumbes constituía la limitación más importante en laderas muy empinadas y muchos podían identificar comunidades afectadas por el problema. El hecho de que la abonera impide el acceso a las milpas durante la temporada de lluvias fue catalogado por el $11 \%$ de los productores como la limitación más relevante. El otro $15 \%$ de los encuestados afirmó que ninguno de los mencionados problemas tenía importancia y que el sistema de abonera no tiene limitaciones graves. Cuando se hizo la recopilación de la segunda desventaja, se notó que la mayoría de los agricultores señalaron como no importantes las otras posibles desventajas que les enseñamos.

La evaluación de los agricultores del sistema de abonera se extiende también al efecto de éste en la calidad de sus parcelas. Se les preguntó si los cambios en la calidad de éstas en el transcurso del tiempo significaban que tendían a mejorar, empeorar o permanecer igual. Las opiniones fueron significativamente diferentes entre los agricultores que practican el sistema de aboneras y los que no. Los primeros perciben con el transcurso del tiempo una mejoría en la calidad de la tierra que atribuyen a las aboneras, mientras que los segundos detectan la degradación que por lo común es consecuencia del cultivo continuo durante períodos largos o de la quema continua. La invasión de malezas, la erosión, el pastoreo y la degradación 
Cuadro 2. Ventajas del frijol de abono.

\begin{tabular}{|c|c|c|c|c|c|}
\hline \multirow[t]{2}{*}{ Característica } & \multicolumn{2}{|c|}{ Primera selección } & \multicolumn{2}{|c|}{ Segunda selección } & \multirow{2}{*}{$\frac{1^{\text {ra }} \text { y } 2^{\text {da }} \text { selección }}{\%}$} \\
\hline & $\begin{array}{c}\text { No. de } \\
\text { agricultores }\end{array}$ & $\%$ & $\begin{array}{c}\text { No. de } \\
\text { agricultores }\end{array}$ & $\%$ & \\
\hline Abono & 32 & 40 & 14 & 18 & 58 \\
\hline Facilita la chapea & 18 & 23 & 21 & 27 & 50 \\
\hline Conserva la humedad & 17 & 21 & 20 & 26 & 47 \\
\hline Controla malezas & 7 & 8 & 18 & 24 & 32 \\
\hline Evita la erosión & 6 & 8 & 3 & 4 & 12 \\
\hline Total & 80 & 100 & 76 & 100 & \\
\hline
\end{tabular}

Cuadro 3. Desventajas del frijol de abono.

\begin{tabular}{|c|c|c|c|c|c|}
\hline \multirow[t]{2}{*}{ Característica } & \multicolumn{2}{|c|}{ Primera selección } & \multicolumn{2}{|c|}{ Segunda selección } & \multirow[t]{2}{*}{$1^{\text {ra }}$ y $2^{\text {da }}$ selección } \\
\hline & $\begin{array}{c}\text { No. de } \\
\text { agricultores }\end{array}$ & $\%$ & $\begin{array}{c}\text { No. de } \\
\text { agricultores }\end{array}$ & $\%$ & \\
\hline Plagas & 37 & 46 & 10 & 12 & 58 \\
\hline Derrumbe & 23 & 28 & 9 & 11 & 39 \\
\hline No son importantes & 12 & 15 & 50 & 62 & 77 \\
\hline Pérdida de primera & 9 & 11 & 12 & 15 & 26 \\
\hline Total & 81 & 100 & 81 & 100 & \\
\hline
\end{tabular}

de la estructura del suelo (tierra dura) son otras causas que citaron del deterioro de las milpas.

La evaluación campesina de los méritos de las aboneras, así como de los efectos a largo plazo que en la calidad de la tierra tienen, resalta un factor central en la amplia aceptación que ha tenido la tecnología: la abonera es congruente con el modelo tradicional de manejo de la tierra. Los agricultores hondureños tienen un conocimiento profundo de los procesos del manejo de la fertilidad, el control de malezas y la conservación de humedad que forman parte del sistema de descanso arbustivo (guamil) que predomina en la región. Las etapas del guamil y las especies que lo componen son bien conocidas, al igual que la función de la sombra en la eliminación de malezas. El maíz de postrera tradicionalmente se siembra sin quemar el rastrojo del cultivo anterior o de otras plantas, para que el mantillo conserve la humedad en el suelo; este conocimiento básico de los productores hondureños les permite entender cómo y por qué funciona la abonera. La evaluación campesina del sistema de abonera en Honduras sugiere que el conocimiento del agricultor de la lógica básica de una práctica o tecnología dada es una condición indispensable para que ésta sea aceptada ampliamente. Por otra parte, la experiencia indica que las nuevas tecnologías que no se basan en el conocimiento campesino requieren un mayor esfuerzo de extensión para poder sobrepasar las limitaciones del conocimiento campesino. 


\section{Fuerzas sociales y económicas}

El contexto social y económico a nivel regional y nacional influye mucho en el desarrollo agrícola. Dos elementos de gran transcendencia para el Litoral Atlántico de Honduras son los fuertes movimientos migratorios que experimenta la región y las políticas de precios del maíz; ambos factores se analizan aquí en forma preliminar.

En las últimas décadas, las laderas del Departamento de Atlántida han sido uno de los principales lugares del país donde se han asentado campesinos desplazados de otras regiones, principalmente el occidente y el interior del país. La región tiene una tasa de crecimiento anual de $4.2 \%$ y, entre 1974 y 1988, la densidad demográfica se ha incrementado de 35 habitantes $/ \mathrm{km}^{2}$ a 56. * Según nuestros datos, cerca del $75 \%$ de los productores encuestados emigraron al Departamento de Atlántida, generalmente en búsqueda de empleo o tierra. Muchos agricultores indicaron también que hay migraciones frecuentes entre las comunidades del Departamento.

Las grandes migraciones permitieron a los agricultores trasladar la tecnología de un lugar a otro. Los datos de la encuesta y las entrevistas informales indican claramente que el sistema de abonera se difundió por todo el Departamento de Atlántida de un agricultor a otro, sin la asistencia de los servicios de investigación y extensión. Entre la gran mayoría de los encuestados, la fuente inicial de información sobre el uso de la abonera fueron sus vecinos o parientes.

El avance de la adopción durante los años 70 sugiere que la divulgación de un agricultor a otro por naturaleza es muy lenta al principio, ya que depende del contacto directo entre éstos. Como ya señalamos, 10 años después de su introducción, solo el 8\% de los agricultores de la región utilizaban aboneras. Sin embargo, este tipo de difusión es muy eficaz una vez que alcanza una cierta "masa crítica" que permite un contacto directo frecuente con la tecnología. Esta situación sugiere una estrategia que permitiría acelerar la difusión de la tecnología en otras zonas con condiciones ambientales y sociales similares, es decir, que los servicios de extensión podrían concentrarse en el establecimiento de una "masa crítica" de adoptadores que posteriormente se encargarían de transferir la tecnología de un agricultor a otro.

Si bien los movimientos migratorios son importantes para la difusión de tecnología, para que se dé la adopción tienen que existir condiciones económicas favorables. En entrevistas informales, los agricultores indicaron que antes de los 1980 había poca comercialización del maíz principalmente porque las laderas del Litoral estaban aisladas tanto de la economía regional como de la nacional. La región se autoabastecía con el maíz de primera y producía poco maíz de postrera. La mejora de las vías de comunicación en la región ha permitido una mayor integración a la economía nacional y ha facilitado la comercialización del maíz.

Tradicionalmente, la producción de maíz en el Litoral Atlántico se concentraba en la producción de primera; sin embargo, en la década de los 80 la importancia de la superficie cultivada en postrera ha crecido en términos relativos a la de primera (Figura 3). De acuerdo con estas cifras, la importancia relativa de la postrera en términos de la superficie cultivada ha crecido a razón de casi $1 \%$ por año en los últimos 15 años. Sin embargo, ese crecimiento parece haberse acelarado en la década de los 80 .

Una de las ventajas que presenta la siembra de postrera sobre la de primera, es que los precios del maíz presentan un patrón estacional que favorece a la producción en esta época del año. Este patrón es una consecuencia de la fuerte estacionalidad de la oferta a nivel nacional, ya que el $80 \%$ de la producción nacional se produce en primera, mientras que sólo el $20 \%$ se produce en postrera. La

* Censo poblacional y del Banco Central, citado en "Proyecto Agricultura en Laderas: Diagnóstico y propuesta preliminar", Consorcio CIAT, IICA, CATIE, 1992. 


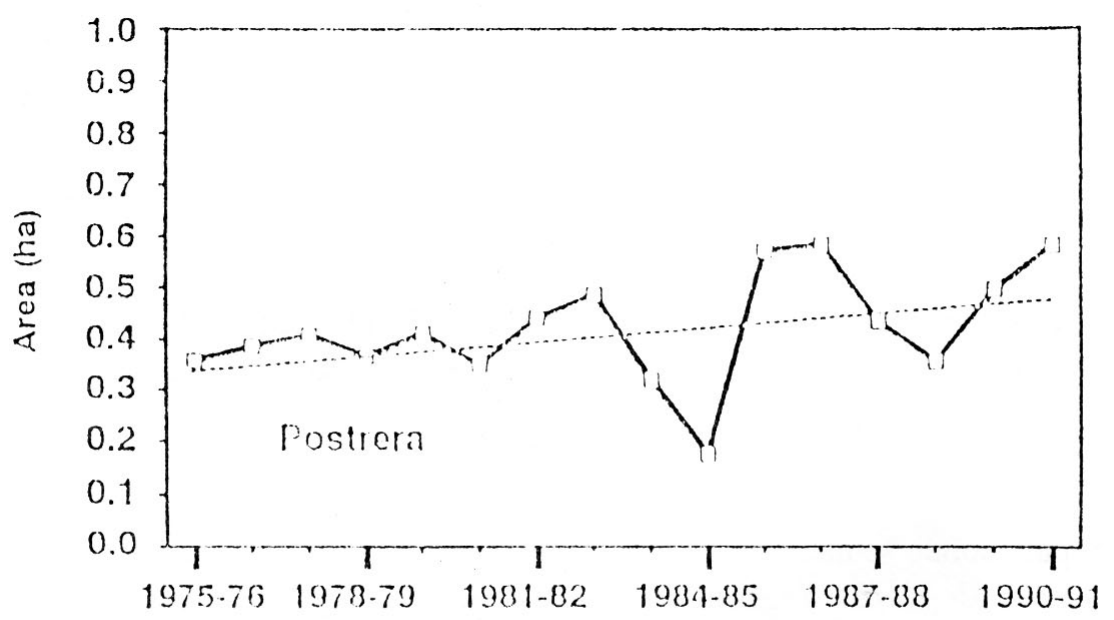

Figura 3. Evolución y tendencia de la impontancia telativa del área culivada en postrera, Litoral Altántico, Honduras, 21975.76 a $199(0) 91$.

estacionalidad en la oferta sumada a un proceso de comercialización sin mucha capacidad de almacenamiento producen una marcada estacionalidad en los precios del maíz. Este factor de mercado sin duda fue importante en el impluso que recibió el proceso de difusión del sistema de abonera, una tecnología bien adaptada a la producción de maíz de postrera.

Otro factor importante que debe ser considerado en una posible explicación del proceso de adopción del sistema de abonera es la rentabilidad del sistema en relación al uso alternativo de la tierra (Cuadro 4) (Sain, et al, 1992). Se calaron la rentabilidad de corto y de largo plazo del sistema de abonera comparándolo con la rotación tradicional de maíz y descanso ( 2 años de maíz y 4 años de descanso). De acuerdo con los resultados obtenidos, el sistema de abonera es rentable a partir del cuarto año cuando se usa el retorno neto al total del capital invertido como medida de rentabilidad de los sistemas. Los primeros tres años deben ser considerados como de inversión. Sin embargo, cuando la rentabilidad se mide de acuerdo a los retornos netos a la mano de obra familiar el sistema de abonera supera al sistema tradicional en el año siguiente a su implantación. Este último resultado es con- gruente con las evaluaciones campesinas del sistema como un ahorrador de mano de obra.

\section{Tenencia de la tierra}

Un elemento considerado clave en la adopción de tecnologías como el sistema de abonera es distinguir entre aquéllos que poseen tierra (propietarios) y los que no la poseen (no propietarios). Se clasifica como propietarios a aquellos agricultores que tanto en primera como postrera declaran alguna actividad realizada en tierra propia. De esa manera, los propietarios pueden también tener parcelas alquiladas o arrendar parte de su propiedad a terceros. Esta definición se refiere sólo al área de finca, independientemente de la tenencia o no de un solar, con el resultado de que los no propietarios pueden o no tener solar.

Según los resultados obtenidos, el $73 \%$ de la población de productores de maíz en el Litoral Atlántico de Honduras sí poseen tierras propias y el 27\% cultiva en tierra ajena. La mayoría de los agricultores de la región, tanto propietarios como no propietarios de tierras agrícolas, poseen un solar donde están asentadas sus casas. 
Cuadro 4. Análisis económico del sistema de abonera y de la rotación tradicional. ${ }^{a}$

\begin{tabular}{|c|c|c|c|c|c|c|}
\hline \multirow{3}{*}{$\begin{array}{l}\text { Tasa de } \\
\text { descuento anual: }\end{array}$} & \multirow{2}{*}{\multicolumn{2}{|c|}{$\begin{array}{c}\text { Tasa marginal } \\
\text { de retorno } \\
\text { de pasar del } \\
\text { sistema tradicional } \\
\text { a la abonera }\end{array}$}} & \multicolumn{4}{|c|}{$\begin{array}{l}\text { Retornos netos a la mano de } \\
\text { obra familiar (L./jor })^{b}\end{array}$} \\
\hline & & & \multicolumn{2}{|c|}{ Abonera } & \multicolumn{2}{|c|}{ Tradicional } \\
\hline & $10 \%$ & $25 \%$ & $10 \%$ & $25 \%$ & $10 \%$ & $25 \%$ \\
\hline \multicolumn{7}{|l|}{ Año } \\
\hline 1 & $-0,81$ & $-0,81$ & $-0,19$ & $-0,19$ & 2,74 & 3,26 \\
\hline 2 & $-1,09$ & $-1,06$ & 3,90 & 3,44 & 3,27 & 3,63 \\
\hline 3 & $-0,41$ & $-0,79$ & 7,16 & 5,83 & 3,27 & 3,63 \\
\hline 4 & 1,31 & 1,70 & 8,48 & 6,49 & 3,27 & 3,63 \\
\hline 5 & 1,24 & 1,36 & 9,04 & 6,55 & 3,27 & 3,63 \\
\hline 6 & 0,99 & 1,07 & 9,25 & 6,36 & 5,45 & 4,28 \\
\hline
\end{tabular}

Un análisis de la relación entre la adopción y la tenencia de la tierra revela una correspondencia significativa entre ambas. Esto se debe a que la abonera requiere un año completo para establecerse. Además, como lo indicaron numerosos agricultores, cuantos más años esté establecida, mejor funciona la abonera. Por esta razón, sería riesgoso para los agricultores que trabajan tierras alquiladas invertir tiempo en establecer aboneras, a menos que tuvieran cierta seguridad de poder seguir cultivando esas tierras en el mediano plazo. Las encuestas en 1982 en Jutiapa y en 1990 a nivel regional también encontraron una relación significativa entre la tenencia de la tierra y la adopción. Por tanto, la tenencia de la tierra parece ser un factor importante en la decisión de adoptar la tecnología.

Pese a lo anterior, la encuesta de 1990 no encontró diferencias en adopción entre los propietarios con dominio pleno (que tienen las escrituras de sus tierras) sobre sus fincas y los que tienen dominio útil (que se han asentado en tierras del dominio público), lo que sugiere que la inseguridad en la tenepcia de la tierra bajo dominio útil no impide que se invierta tiempo y dinero en el establecimiento de aboneras.
La función de la tenencia de la tierra en la adopción del sistema de abonera se comprobó mediante un análisis de la desadopción de la tecnología. Como ya se señaló, cerca del $16 \%$ de los agricultores encuestados han utilizado aboneras en algún momento en el pasado, pero actualmente no siembran el maíz de postrera en esa forma. No obstante, los aspectos negativos de la tecnología no parecen haber influido en su decisión de desadoptarla. Cuando se les preguntó la razón que los motivó a abandonar la práctica, la respuesta más frecuente fue la falta de acceso a tierra propia (Cuadro 5).

Cuadro 5. Razones de haber abandonado el sistema de abonera.

\begin{tabular}{lcc}
\hline Razones & $\begin{array}{c}\text { No. de } \\
\text { agricultores }\end{array}$ & $\%$ \\
\hline Falta de tierra propia & 12 & 60 \\
Abonera destruida por fuego & 3 & 15 \\
Vendió terreno & 2 & 10 \\
Cambió de cultivo & 2 & 10 \\
No hay cosecha de primera & 1 & 5 \\
\hline Total & 21 & 100 \\
\hline
\end{tabular}


Estos datos sugieren que las tendencias futuras en la difusión de tecnología probablemente serán afectadas por los modos de distribución de la tierra. La tasa de adopción $(66 \%)$ se acerca el porcentaje de agricultores con tierras propia.s (73\%), lo que probablemente explique en parte la tasa de adopción más lenta observada en años recientes (Figura 2). Sin embargo, hay que tomar en cuenta que ya se ha desarrollado en la zona un mercado de arrendamiento de aboneras, lo cual permite a los agricultores sin tierra tener acceso a ellas. Por tanto, si bien la tenencia de la tierra es un factor que limita la adopción de la tecnología, la evolución de los mercados de arrendamiento de aboneras quizá eleve las tasas de adopción por encima de los niveles de propiedad de tierra.

\section{Tamaño de la finca}

Dado que en el sistema de abonera se cultiva el maíz de primera en un campo separado de la abonera, sería lógico pensar que se requieren fincas más grandes para permitir la rotación (Cuadro 6). Los datos de la encuesta indican que hay una relación significativa entre la adopción y el tamaño de la finca. El grupo de adoptadores tienen en promedio $14.3 \mathrm{mzs}$ de finca (incluyendo la tierra propia y la alquilada o prestada), mientras que los agricultores que no han adoptado la tecnología tienen un promedio de 5.5 mzs. Al parecer, hay una mayor adopción del sistema de aboneras entre los agricultores con fincas de mayor tamaño. No obstante, la relación entre la adopción y el tamaño de la finca está fuertemente condicionada por la forma de tenencia de la tierra. Entre los propietarios, los que han adoptado el sistema tienen en promedio propiedades más grandes que los que no lo han adoptado (16,5 mz comparadas con $8,5 \mathrm{mz}$, respectivamente), pero estas diferencias no son estadísticamente significativas. Así pues, si bien los productores que tienen fincas más grandes se encuentran en mejor posición de adoptar la tecnología, es más importante el hecho de poseer tierra.

No obstante, el tamaño de la finca no es un limitante absoluto en cuanto a la adopción del sistema de abonera, puesto que el $56 \%$ de los agricultores propietarios de menos de $2 \mathrm{mz}$ han adoptado la tecnología, un porcentaje que, si bien es inferior a los observados en otros grupos de agricultores, no deja de ser elevado.

El análisis de la relación entre la adopción del sistema de abonera y la tenencia de la tierra y el tamaño de la finca que presentamos arriba debe tomar en consideración la abundancia relativa de tierra en descanso en el Departamento de Atlántida, así como el bien desarrollado mercado de arrendamiento de tierra. Los que no poseen tierra y los agricultores que tienen fincas muy pequeñas pueden alquilar tierra a un precio bajísimo bajo el sistema de descanso arbustivo, en especial para el maíz de primera, de los ganaderos que desean convertir la tierra en descanso en pastos. De esta manera, esos agricultores pueden dedicar sus pequeñas parcelas a la rotación maíz frijol de abono. En resumen, es el sistema social que afecta el acceso

Cuadro 6. Tamaño de la finca entre los propietarios que adoptaron y no adoptaron el sistema de abonera.

\begin{tabular}{cccccc}
\hline & \multicolumn{2}{c}{ Utiliza aboneras } & & \multicolumn{2}{c}{ No utiliza aboneras } \\
\cline { 2 - 3 } \cline { 5 - 6 } $\begin{array}{c}\text { Tamaño } \\
\text { de finca }\end{array}$ & $\begin{array}{c}\text { No. de } \\
\text { agricultores }\end{array}$ & $\%$ & & $\begin{array}{c}\text { No. de } \\
\text { agricultores }\end{array}$ & $\%$ \\
\hline $0.5-2$ & 5 & 55.6 & & 4 & 44.4 \\
$2-5$ & 19 & 76.0 & & 6 & 24.0 \\
$5-10$ & 17 & 70.8 & & 7 & 29.2 \\
$>10$ & 31 & 86.1 & & 5 & 13.9 \\
\hline
\end{tabular}


a la tierra el que determina las posibilidades para la difusión de la tecnología, y no el tamaño de la finca ni la forma de tenencia de la tierra.

\section{CONCLUSIONES Y RECOMENDACIONES}

La difusión y amplia adopción del sistema de abonera en el Litoral Atlántico de Honduras constituye un avance que tiene consecuencias significativas para el manejo del maíz en laderas en el trópico húmedo de Centroamérica y México. En el presente trabajo, se documentan los aspectos fundamentales de ese sistema y se identifican algunas condiciones que determinan la adopción de la tecnología.

Se difundió de un agricultor a otro, sin la asistencia de los servicios de extensión nacionales o privados, hasta llegar a la mayoría de las comunidades en las laderas de Atlántida. En la actualidad, se estima que el 66\% de los agricultores en ladera en ese Departamento cultivan el maíz de postrera en aboneras. La rapidez y uniformidad con que se establecieron las aboneras demuestran que la difusión de un agricultor a otro puede ser muy eficaz. Los servicios de extensión de la región pueden aprovechar la eficacia de las redes campesinas de comunicación concentrando sus esfuerzos en grupos estratégicos de agricultores en las aldeas y promoviendo el intercambio de información entre ellos.

Los agricultores hondureños conocen bien las ventajas y desventajas del sistema de abonera. Si bien consideran que los efectos fertilizantes de la leguminosa son la razón más importante de usar la tecnología, reconocen que existen también otros beneficios como el ahorro de la mano de obra, la conservación de la humedad en el suelo y el control de la erosión. El alto valor que se da al ahorro de la mano de obra revela la importancia de ésta en las decisiones de los campesinos respecto a las opciones tecnológicas. Es claro que los agricultores de escasos recursos de Atlántida valoran las tecnologías que permiten grandes ahorros de mano de obra, pues asignan un peso considerable a este aspecto. Por otra, al considerar las tecnologías orientadas a conservar los recursos naturales que son recomendables para los agricultores de escasos recursos, cabe recordar que las que requieren mucha mano de obra no serán bien recibidas por ellos.

Las evaluaciones técnica y campesina indican que la abonera es congruente con el modo tradicional de manejar el suelo, las malezas y el agua en el cultivo ambulatorio, un hecho útil para promover una adopción rápida y extensa. Este hallazgo sugiere que la generación y difusión de nuevas tecnologías en la región mejoraría si los investigadores comprendieran las características y deficiencias de los conocimientos campesinos. En esta forma, podrían generar tecnologías que toman esos conocimientos como punto de partida, lo cual facilitaría la subsecuente difusión de tecnología. Cuando esto no sea posible, los servicios de extensión pueden difundir información educativa en las zonas en que los conocimientos de los campesinos son limitados.

Es mucho más probable que los agricultores propietarios adopten la tecnología que los campesinos que trabajan tierras arrendadas. Las formas de arrendamiento suelen ser demasiado inseguras para justificar que los agricultores sin tierras establezcan aboneras. Sin embargo, esto no significa que la seguridad a largo plazo que brinda el ser propietario sea una condición indispensable para adoptar la tecnología; los agricultores que tienen dominio útil como los que tienen tierras oficialmente escrituradas. El análisis económico y las entrevistas celebradas en la región sugieren que un horizonte de planificación de mediano plazo (tres o cuatro años) compensa con creces la inversión inicial en el establecimiento de la abonera.

Hay un mayor nivel de adopción entre los agricultores que poseen fincas más grandes. No obstante, un número significativo de agricultores con menos de 2 hectáreas las adoptan también. Estos resultados sugieren que el tamaño de la finca no es un factor limitante absoluto para la adopción del sistema de abonera. 
Pese a que la propiedad de la tierra y el tamaño de la finca son sólo limitaciones parciales de la adopción en Atlántida, es necesario tener en cuenta la abundancia relativa de tierra en descanso y el bien desarrollado mercado de arrendamiento en la región. En resumen, el factor que determina la difusión de la tecnología suele ser el sistema social que afecta el acceso a la tierra, no el tamaño de la finca ni los modos de tenencia de la tierra. En consecuencia, es necesario hacer un examen minucioso de los patrones de uso de la tierra a fin de evaluar el potencial de las aboneras en una región más extensa.

La investigación agrícola tiene una función primordial en el desarrollo de otras tecnologías de abono verde en los sistemas de maíz en ladera, tanto en Atlántida como en otros lugares. La posible intensificación del sistema mediante la introducción del cultivo intercalado de leguminosas con maíz de primera constituye un importante tema de investigación (Zea, 1990-1991-1992). Quizá la lección más valiosa que nos enseña el uso y difusión del frijol de abono en Atlántida sea que los abonos verdes pueden ser elementos eficaces y adoptables en los sistemas de cultivo en ladera. Lo más importante son los principios del abono verde que aplican los campesinos, no la leguminosa ni la práctica de cultivo. Así pues, las innovaciones ideadas por los agricultores de escasos recursos nos señalan una prometedora área de investigación.

\section{AGRACECIMIENTO}

El presente trabajo enfatiza el análisis socio-económico del uso de frijol de abono en Atlántida, basado en una versión mas extendida del mismo trabajo. Cita Correcta: D. Buckles, 1. Ponce, G. Sain y G. Medina. 1992. Tierra cobarde se vuelve valiente: Uso y difusión del frijol de abono (Mucuna deeringiana) en las laderas del Litoral Atlántico de Honduras. México, D.F.: CIMMYT. Este documento fue desarrollado bajo los auspicios del Programa Regional de Maíz para Centroamérica y el Caribe (PRM), una red internacional de investigación conjunta, que hace énfasis en la generación de germoplasma mejorado de maíz, investigación agronómica para la producción sostenible de maíz y estudios socioeconómicos para evaluar la adopción y efecto de la tecnología en los sistemas de producción. Los autores desean agradecer la participación en el levantamiento de la encuesta presentada en este documento de Marlon Arita, Helington Antúnez, Heber Bojorque, Armando Borjas, Ignacio Cortés, Roberto Escoto, Dagoberto Flores, Carmen Regina García Hiza, Melesio Guillén, Gustavo López, María Gricel Navarro, José María Reina, Oscar A. Robles, Carlos Guillermo Rosales G. y Jesús Zelaya. Se hace patente el agradecimiento por el especial apoyo brindado por Héctor Nolasco, Director Regional de la Secretaría de Recursos Naturales en La Ceiba, Atlántida, la Agencia de Desarrollo en Tela y en Jutiapa y la Dirección del Proyecto Desarrollo del Bosque Latifoliado (PDBL).

\section{LITERATURA CITADA}

PNIA-CIMMYT. (Gua.). PROGRAMA DE MAIZ, 1983. "Informe de la encuesta formal en el área de Jutiapa", manuscrito inédito. Guatemala.

BUCKLES, D. 1990. "Resultados de la encuesta exploratoria sobre el uso de frijol de abono (Stizolobium deeringianum) en laderas del Litoral Atlántico de Honduras". En: Análisis de los Ensayos Regionales de Agronomía, 1991, Programa Regional de Maíz para Centroamérica, Panamá y El Caribe.

BUCKLES, D.; PERALES, H. 1992. "Farmer Experimentation with Green Manures in the Sierra de Santa Marta, V eracruz", documento inédito, CIMMYT -PSSM.

BUCKLES, D.; PONCE, 1.; SAIN, G.; MEDINA, G. 1991. "Resultados de la encuesa exploratoria sobre el uso de frijol de abono (Stizolobium deeringianum) en laderas del Litoral Atlántico de Honduras". En: Programa Regional de Maíz para Centro américa y el Caribe, 1992. Análisis de los Ensayos Regionales de Agronomía. Guatemala, Guatemala.

DURON, F. 1989. Avances sobre investigación de abonos verdes en el Litoral Atlántico de Honduras". En: Proyectos Colaborati vos de Agronomía, desarrollo y mejoramiento de germoplasma en maíz (Zea mays L.). 1990. Programa Regional de Maíz para Centroamérica, Panamá y el Caribe. 
SAIN, G.; PONCE, I; BORBON, E. 1992. "Rentabilidad del sistema de abonera en el litoral Atlántico de Honduras", presentado en: A Workshop on Slash/Mu1ch Practices, CA TIE, Turrialba, Costa Rica, Octubre. p. 12-16.

ZEA, J. L. 1990. "Efecto de intercalar leguminosas a diferentes dosis de fósforo sobre el rendimiento de maíz. (Zea mays L.), en 24 ensayos a través de Centroamérica. En: "Análi- sis de los ensayos regionales de agronomía. Programa Regional de Maíz para Centroamérica, Panamá y el Caribe, 1991. Guatemala.

ZEA, J. L. 1993. Efecto de intercalar leguminosas sobre el rendimiento de maíz (Zea mays L.) en nuevas localidades de Centroamérica. Agron. Mesoam. 4:18-22. 